\title{
ESPECIAL
}

\section{¿Es la ética profesional un lujo del que podemos prescindir?} \author{
María Chiva ${ }^{a}$, Josep Lluch ${ }^{a, b}{ }^{a,}$ Jorge Martín $^{a}$, Alma Palau ${ }^{a}$, Aitor Sánchez ${ }^{a, c}$,
Eva María Trescastro-López
}

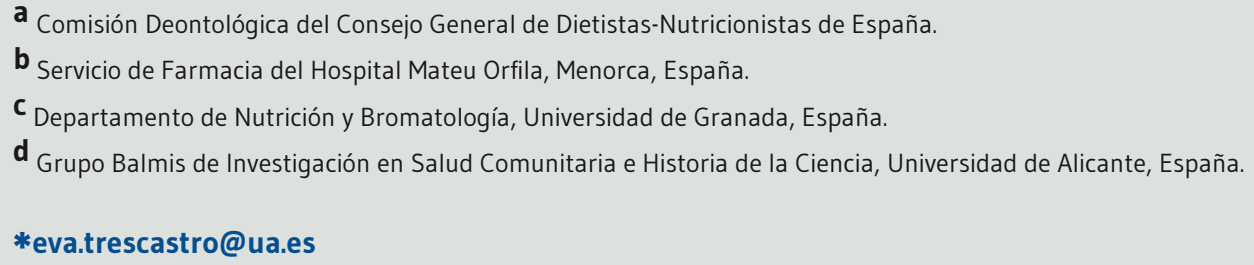

Recibido el 3 de diciembre de 2014; aceptado el 25 de febrero de 2015.

\section{$\sum$ ¿Es la ética profesional un lujo del que podemos prescindir?}

\section{PALABRAS CLAVE}

Dietista-Nutricionista;

Códigos de ética;

Ética;

Deontología;

Ética profesional;

Formación continua;

Programa de estudios.

\section{RESUMEN}

En una sociedad como la actual donde la recuperación y la educación en valores está siendo reivindicada, la ética profesional adquiere un protagonismo y una importancia fundamental y la formación de los futuros profesionales aparece como una de las cuestiones clave. En este trabajo se han analizado en primer lugar los contenidos de ética en las Universidades españolas donde se estudia el Grado en Nutrición Humana y Dietética y los contenidos de ética en las dobles titulaciones. En segundo lugar se han analizado los resultados de la encuesta online que se realizó entre el 1 y el 11 de septiembre de 2014 en donde se incluía una pregunta sobre ética profesional y, por último, se ha tomado el Código Deontológico de los DietistasNutricionistas españoles como ejemplo para resolver algunas de las cuestiones que están surgiendo en nuestra profesión relacionadas con el patrocinio de la industria. En conclusión, sería recomendable que la asignatura de ética/bioética o deontología estuviera en 4ำ curso y que en las Universidades donde se imparte doble titulación se incluyan contenidos propios de nutrición humana y dietética. Debemos promover la creación de una cultura deontológica propia y específica de la nutrición humana y la dietética que empieza con la formación universitaria y continúa con la formación a lo largo de la vida profesional.
\end{abstract}


Is professional ethics a luxury we can do without?

KEYWORDS

Dietetic-Nutritionist;

Codes of ethics;

Ethics;

Ethical theory;

Ethics professional;

Education

continuing;

Curriculum.

\begin{abstract}
In today's society where the recovery and education of values is being reclaimed, professional ethics is gaining an essential importance, and the education of future professionals is becoming one of the key questions. First of all, this project analyses the content of ethics based on Spanish universities where the Human Nutrition and Dietetics Undergraduate Degree is taught, as well as the content of ethics of dual undergraduate programmes. In second place, the results of an online survey have been analysed. This survey, carried out between 1 and 11 September 2014, included a question on professional ethics and, finally, the Spanish Code of Ethics for Dieticians and Nutritionists was used as an example to solve doubts that were raised regarding our profession related to the patronage of the industry. To conclude, it would be recommendable for the ethics/ bioethics subject to be given in the fourth year of the undergraduate degree and for universities where there is a dual undergraduate programme to include content from both human nutrition and dietetics. We should promote the creation of our own ethical culture specifically for human nutrition and dietetics that starts at the university education stage and continues in training throughout the whole professional life.
\end{abstract}

\section{CITA}

Chiva M, Lluch J, Martín J, Palau A, Sánchez A, Trescastro-López EM. ¿Es la ética profesional un lujo del que podemos prescindir? Rev Esp Nutr Hum Diet. 2015; 19(3): 175 - 183. DOI: 10.14306/renhyd.19.3.138

\section{INTRODUCCIÓN}

En una sociedad como la actual donde la recuperación y la educación en valores está siendo reivindicada, la ética profesional adquiere un protagonismo y una importancia fundamental en desarrollo de la misma, y la formación de los futuros profesionales aparece como una de las cuestiones clave.

Para intentar responder a la pregunta que da título al artículo, en primer lugar se va a analizar la formación universitaria en materia de ética/bioética o deontología que reciben los Dietistas-Nutricionistas (D-N) de España. En segundo lugar, se van a analizar los resultados de la encuesta que se realizó a través de un cuestionario on-line entre el 1 de septiembre y el 11 de septiembre de 2014 a los miembros del Consejo General de Dietistas-Nutricionistas de España (CGD-NE) y a los amigos de la Fundación Española de Dietistas-Nutricionistas (FEDN)². Por último se abordará a modo de ejemplo, el principio nํㅡㄴ 20 del Código Deontológico de los Dietistas-Nutricionistas españoles ${ }^{3}$, que afirma que un D-N
"No solicitará, aceptará u ofrecerá regalos, incentivos económicos o de otra consideración que afecten o den apariencia razonable de afectar el criterio profesional". Este punto guarda una estrecha relación con alguna de las cuestiones que se reivindican por parte de la iniciativa "Dietética sin Patrocinadores"4.

\section{CONTENIDOS DE ÉTICA EN LAS CONTENIDOS DE ÉTICA EN LAS UNIVERSIDADES ESPAÑOLAS DONDE SE ESTUDIA EL GRADO EN NUTRICIÓN HUMANA Y DIETÉTICA}

La Orden CIN/730/2009, de 18 de marzo, por la que se establecen los requisitos para la verificación de los títulos universitarios oficiales que habilitan para el ejercicio de la profesión de D-N, establece que entre las competencias que los estudiantes deben adquirir, está la de "Reconocer los elementos esenciales de la profesión del $D-N$, incluyendo los principios éticos, responsabilidades legales y el ejercicio de la profesión, aplicando el principio de justicia social a la práctica profesional 
y desarrollándola con respeto a las personas, sus hábitos, creencias y culturas $^{\prime \prime}$. Como bien señala este punto, es primordial formar a los estudiantes tanto en valores como en ética, ya que como profesionales sanitarios tendrán una gran responsabilidad en el estado de salud del individuo y/o de la comunidad.

Para abordar la primera de las cuestiones se va a analizar si en las Universidades españolas se imparte sólo el Grado en Nutrición Humana y Dietética (NHyD) o si se imparten dobles titulaciones y si el hecho de estudiar una doble titulación puede influir de forma directa en la formación en ética/bioética o deontología que reciben los estudiantes e indirectamente en la ética profesional.

En la actualidad, en España se puede estudiar el Grado en Nutrición Humana y Dietética en 23 Universidades ${ }^{6-36}$ (Figura 1), siendo 14 de ellas públicas (61\%) y 9 privadas (39\%). La última fecha de búsqueda ha sido el 6 de octubre de 2014.
Del total de 23 Universidades donde se puede obtener el título de Graduado/a en NHyD, 8 de ellas (34\%) ofertan dobles titulaciones $8,9,11,13,14,16,17,25,29,34$ (Tabla 1 ), además del propio Grado.

En todas las Universidades existe una asignatura relacionada con la ética/bioética o deontología y en la mayoría de ellas se tratan aspectos tan importantes como: códigos deontológicos, ética profesional, Codex Alimentario, relaciones con el paciente/cliente, diversos aspectos legislativos, etc., pero sólo algunas de ellas incluyen en su temario el Código Deontológico del D-N: Universidad de Alicante ${ }^{19}$, Universidad de Navarra ${ }^{24,25}$ y Universidad de Zaragoza ${ }^{32}$.

Respecto al nํ de créditos de la asignatura, la distribución es variable (Figura 2).

Respecto al curso en el que se encuentra la asignatura dentro del plan de estudios, la distribución se puede observar en la Figura 3.

Figura 1. Universidades españolas donde estudiar el Grado en Nutrición Humana y Dietética.

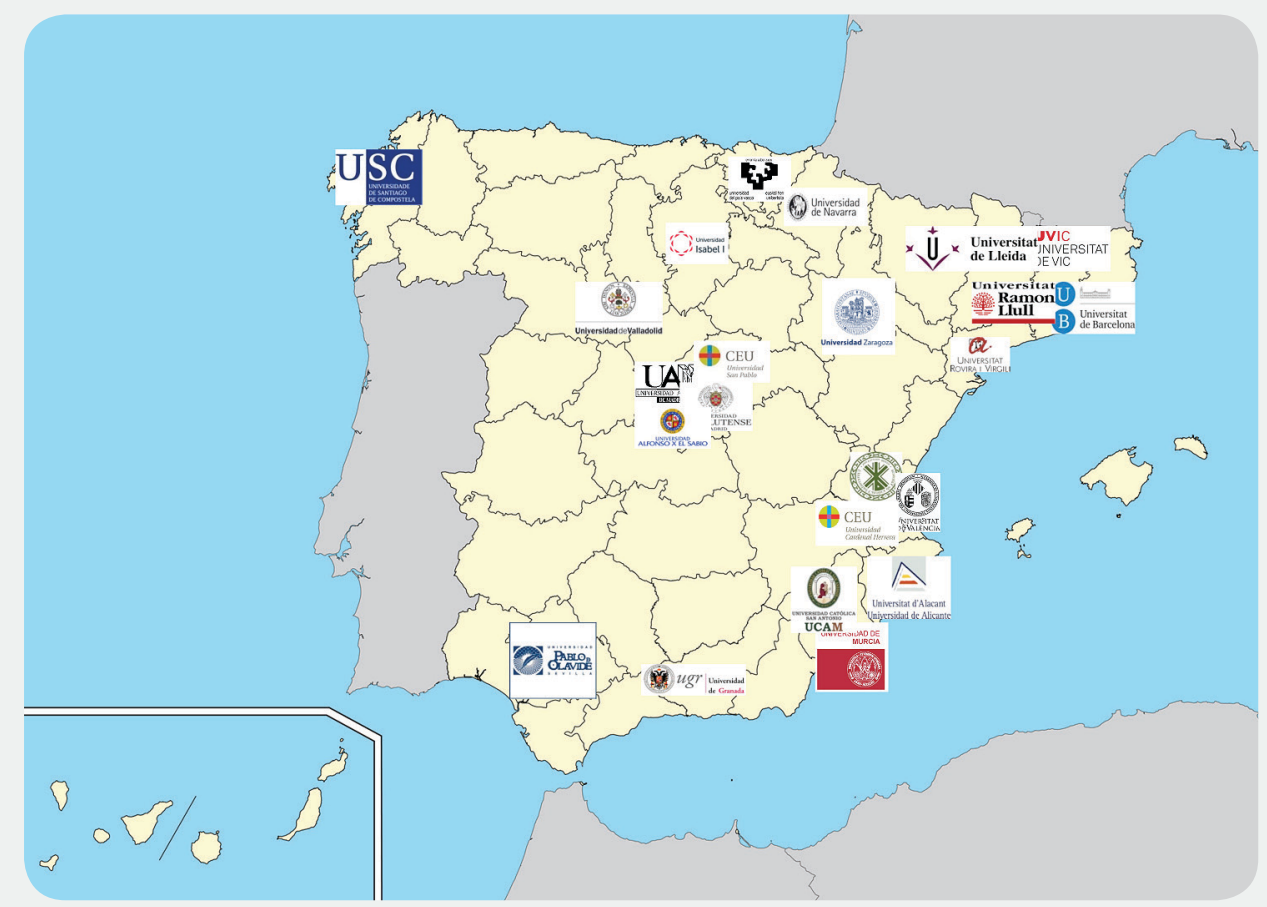

Fuente: elaboración propia. 
Rev Esp Nutr Hum Diet. 2015; 19(3): 175 - 183

¿Es la ética profesional un lujo del que podemos prescindir?

Tabla 1. Universidades donde se puede estudiar doble grado.

\begin{tabular}{|c|c|c|c|}
\hline Universidad & Grado NHyD & & Otra titulación \\
\hline $\begin{array}{c}\text { Universidad Cardenal Herrera CEU } \\
\text { (privada) }\end{array}$ & NHyD & + & Farmacia \\
\hline $\begin{array}{l}\text { Universidad Católica de Valencia San } \\
\text { Vicente Mártir (privada) }\end{array}$ & NHyD & + & Enfermería \\
\hline Universidad de Navarra (privada) & NHyD & + & Farmacia \\
\hline $\begin{array}{l}\text { Universidad Católica San Antonio de } \\
\qquad \text { Murcia (privada) }\end{array}$ & NHyD & + & Ciencia y Tecnología de los Alimentos \\
\hline \multirow[t]{2}{*}{ Universidad Alfonso X el Sabio (privada) } & NHyD & + & Farmacia \\
\hline & NHyD & + & Ciencias de la Actividad Física y el Deporte \\
\hline \multirow[t]{2}{*}{ Universidad San Pablo CEU (privada) } & NHyD & + & Farmacia \\
\hline & NHyD & + & Enfermería (pendiente de aprobación por ANECA) \\
\hline $\begin{array}{l}\text { Universidad Internacional Isabel I de } \\
\text { Castilla (privada on-line) }\end{array}$ & NHyD & + & Ciencias de la Actividad Física y el Deporte \\
\hline Universidad de Valencia (pública) & NHyD & + & Farmacia \\
\hline
\end{tabular}

Fuente: elaboración propia.

Figura 2. Créditos de la asignatura de ética/bioética o deontología.

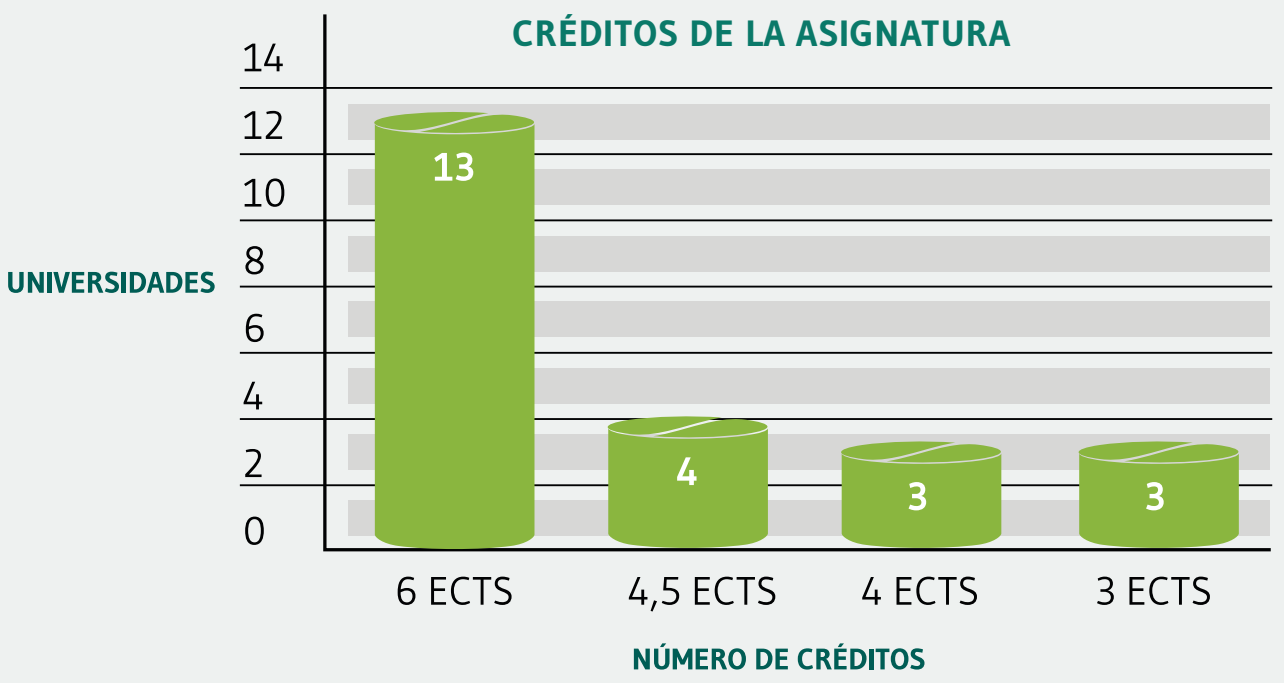

Fuente: elaboración propia. 
Figura 3. Curso en el que se imparte la asignatura de ética/bioética o deontología.

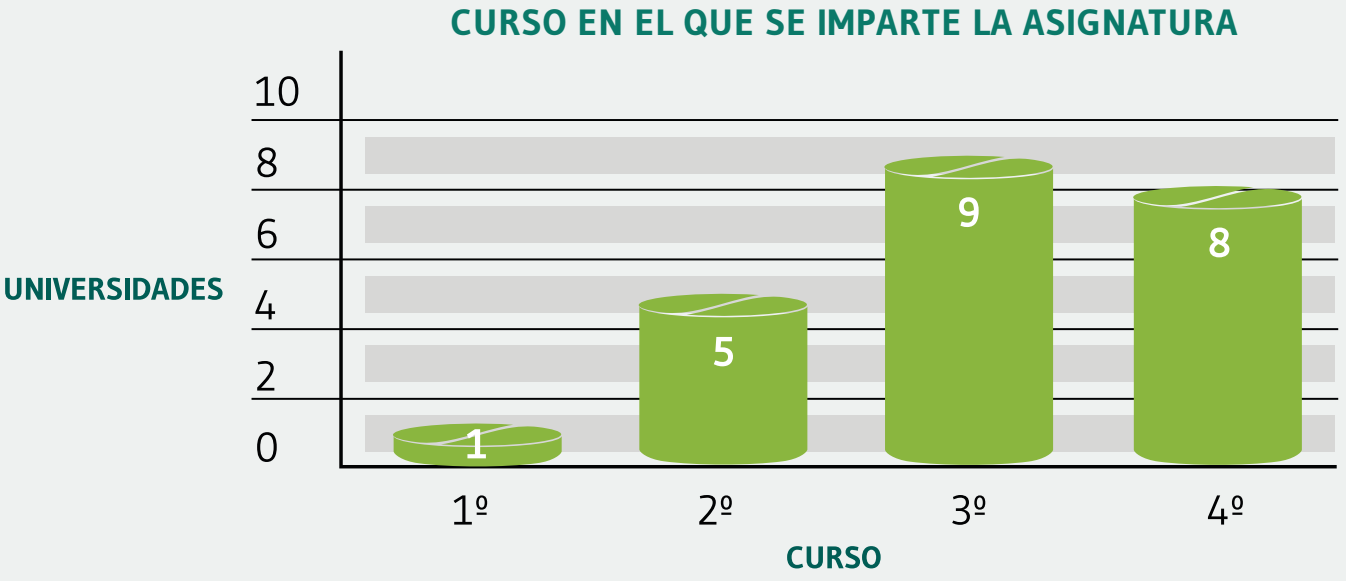

Fuente: elaboración propia.

\section{CONTENIDOS DE ÉTICA EN LAS DOBLES TITULACIONES}

A continuación se ha procedido a analizar los contenidos de las asignaturas de ética/bioética o deontología en las dobles titulaciones y se ha observado que los contenidos pueden ser diferentes si se estudia sólo el Grado en NHyD o si se estudia una doble titulación. En este último caso, el Grado de NHyD se "suma" al previo existente, de manera que los contenidos que se imparten en estas asignaturas no son específicos de la nutrición humana y la dietética. En este sentido, hay que señalar que aunque se comparten contenidos similares en ambas profesiones, en algunas de las dobles titulaciones no se incluyen referencias bibliográficas específicas de nutrición, ni legislación alimentaria, ni Codex Alimentario, etc.

En la Universidad Alfonso X el Sabio, la asignatura de ética/bioética o deontología es diferente si se trata del Grado en NHyD o del doble Grado "Farmacia + NHyD", en donde los contenidos que se incluyen son exclusivamente de Farmacia8.

En la Universidad Cardenal Herrera de Valencia, la asignatura que se imparte en el doble Grado "NHyD + Farmacia" Ileva por título "Historia, Legislación y Deontología Farmacéutica"14.
En la Universidad Católica de Valencia San Vicente Mártir, donde se imparte el doble Grado "NHyD + Enfermería", dicha asignatura pertenece al plan de estudios de Enfermería y sus contenidos son únicamente de Enfermería ${ }^{11}$.

En la Universidad CEU San Pablo de Madrid, la asignatura es diferente si se imparte en el doble Grado "NHyD + Farmacia", pasando a llamarse en la doble titulación "Legislación y Deontología farmacéutica"16. En el caso del doble Grado "NHyD + Enfermería" no existe ninguna asignatura específica de ética/bioética o deontología (el plan de estudios de Enfermería tiene un Modifica ante ANECA pendiente de aprobación) ${ }^{17}$.

En la Universidad Internacional Isabel I de Castilla no aparecen contenidos de la asignatura, y en el doble grado "NHyD + Ciencias de la Actividad Física y el Deporte (CAFD)", la asignatura se denomina "Valores sociales y deontología profesional" $\mathrm{y}$ corresponde al plan de estudios de CAFD ${ }^{34}$.

Estos datos que se acaban de mostrar relativos a la formación académica son básicamente descriptivos, ya que no se puede entrar en el detalle de los contenidos de todas y cada una de las asignaturas, aspecto formativo que obviamente va a influir en la ética profesional. 


\section{$r$} ENCUESTA SOBRE ÉTICA PROFESIONAL

Entre el 1 de septiembre y el 11 de septiembre de 2014 se llevó a cabo a través de la web una encuesta a todos los D-N del CGDNE y amigos de la FEDN. La encuesta se envió a 2689 personas y se obtuvieron un total de 347 respuestas (12,9\%).

De estas 347 personas, sólo se le realizó la pregunta relacionada con la ética profesional a aquellas que estaban ejerciendo, que representaban un total de 241 ejercientes.

"Durante el ejercicio de la profesión, ¿te ha surgido algún conflicto deontológico?"

- Respecto a los estándares de salud

- Respecto a las leyes y reglamentos

- Respecto al Código Deontológico y Buenas Prácticas

- Respecto al ejercicio de la profesión

- Respecto al trato con los pacientes/clientes
- Respecto a tus capacidades personales

- Respecto a los colegas y otros profesionales

- Nunca me ha surgido un conflicto deontológico

- Otro

De todas las respuestas, 139 personas $(57,7 \%)$ respondieron que "Nunca les había surgido un conflicto deontológico" y 102 personas (42,3\%) sí manifestaron haber tenido algún conflicto deontológico (Figura 4).

\section{EL CóDIGO DEONTOLóGICO COMO EJEMPLO}

En la actualidad, en el seno de nuestra profesión están surgiendo iniciativas que reivindican el ejercicio profesional sin ningún tipo de sponsor, como es el caso de "Dietética sin Patrocinadores" ${ }^{4}$. En este caso conviene consultar el principio n 20 del Código Deontológico ${ }^{3}$ recogido en el apartado de

Figura 4. Diagrama de flujo.

Dietistas-Nutricionistas miembros del CGD-ND y amigos de la FEDN a

los que se les envía la encuesta

$$
(n=2.689)
$$

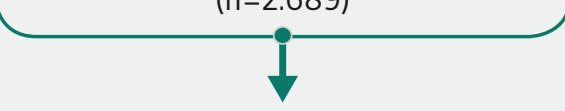

№ de respuestas de la encuesta $(n=347)$

Dietistas-Nutricionistas no ejercientes $(n=106)$
Dietistas-Nutricionistas a los que sí les ha surgido algún conflicto ético $(n=102)$
Dietistas-Nutricionistas a los que nunca les ha surgido ningún conflicto ético $(n=139)$

Fuente: elaboración propia. 
Responsabilidades hacia la Profesión y que guarda relación con estas cuestiones.

Este principio afirma que un D-N "No solicitará, aceptará u ofrecerá regalos, incentivos económicos o de otra consideración que afecten o den apariencia razonable de afectar el criterio profesional."

De este principio se derivan 3 buenas prácticas (BP):

- BP1: No será una violación de este principio para el D-N aceptar compensación como consultor o empleado o como parte de una beca de investigación o programa de patrocinio de las empresas, siempre y cuando la relación sea abiertamente declarada y el D-N actúe con integridad en el desempeño de los servicios o responsabilidades.

- BP2: Este principio no se opone a que un D-N acepte regalos de valor nominal, atienda programas educativos, comidas en el marco de intercambios de la información educativa, muestras gratuitas de productos o artículos similares, siempre y cuando estos artículos no se ofrezcan a cambio de o con la expectativa de, y no den lugar a conductas o servicios que sean contrarios al criterio riguroso del D-N.

- BP3: La prueba de una apariencia impropia dependerá de si dicha conducta pudiera crear en personas razonables la percepción de que el D-N no es capaz de ejercer sus responsabilidades profesionales con integridad, imparcialidad y su competencia está afectada.

En el caso de que exista una denuncia por incumplimiento del Código Deontológico o se desee hacer una consulta al respecto, parece oportuno que sea la Comisión Deontológica Nacional la encargada de analizar y decidir sobre estas cuestiones: porque el Código Deontológico es una guía general para el ejercicio profesional y no puede recoger con detalle situaciones que precisen actuaciones concretas.

El Código Deontológico y la Comisión Deontológica tienen tanto la misión de proteger al profesional de sus propias flaquezas, como de guiarle en las acciones profesionales frente a la sociedad, cliente/paciente, colegas y otros profesionales para que actúe siempre en el marco de la evidencia científica y con la humanidad que requieren todos los temas relacionados con la alimentación y nutrición humana ${ }^{3}$. Así mismo, la Comisión Deontológica debe promover que existan cuestiones relacionadas con la ética profesional en las reuniones científicas y debe elaborar documentos de consenso.

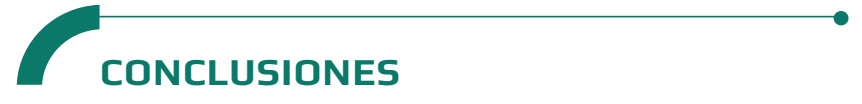

Respondiendo a la pregunta que nos planteamos en el inicio, ¿Es la ética profesional un lujo del que podemos prescindir? la respuesta está clara: no. No podemos prescindir de ella, porque solamente con una buena formación y quehacer diario, lograremos el objetivo primordial de elevar el estándar de la práctica y la buena praxis profesional.

Sería recomendable que la asignatura de ética/bioética o deontología estuviera en $4^{\circ}$ curso, cuando los estudiantes han recibido ya la mayor parte de la formación, han madurado personal y profesionalmente y son capaces de aprovechar esta asignatura al máximo. Además, en aquellas Universidades donde se imparte doble titulación se deberían incluir contenidos propios de la nutrición humana y la dietética.

A la luz de los datos expuestos es importante insistir en la importancia de la formación universitaria en materia de ética profesional, con el fin de crear una cultura deontológica que facilite la resolución de situaciones profesionales conflictivas y pueda ayudar a desvelar y discernir los momentos éticos en la práctica profesional; en definitiva proporcionar al futuro profesional las herramientas para abordar posibles conflictos. Hablar de ética es tratar sobre cómo se debe actuar, es hablar de valores que deben ser defendidos y respetados, planteándose en ocasiones dilemas que exigen respuestas responsables. Actuar éticamente tiene que ver con la justificación y con las razones que soportan la decisión adoptada. Queda claro, por tanto, que no bastan las buenas intenciones; los comportamientos y sus resultados también deben orientarse de acuerdo con principios y valores sólidos ${ }^{37}$.

Debemos promover la creación de una cultura deontológica propia y específica de la nutrición humana y la dietética que empieza con la formación universitaria, pero que no acaba aquí y continúa en la formación a lo largo de la vida profesional. Debemos ser capaces de incorporar en el trabajo diario esta dimensión deontológica ya que sólo así conseguiremos diferenciarnos ante los demás profesionales y ante la sociedad como verdaderos expertos en nutrición humana y dietética.

\section{AGRADECIMIENTOS}

Este trabajo se ha desarrollado en el marco de la Beca Predoctoral del Programa de Formación de Profesorado Universitario (Ref.: FPU/ AP2010-0593) del Ministerio de 
Educación y del Programa Prometeo de la Generalitat Valenciana para grupos de investigación de excelencia (Ref.: Prometeoll/2014/015).

\section{CONFLicto de INTERESES}

Los autores manifiestan la no existencia de conflictos de intereses al redactar el manuscrito.

\section{$\longrightarrow$ \\ BIBLIOGRAFÍA}

1. Consejo General de Dietistas-Nutricionistas de España [portal en internet]. Valencia: Consejo General de DietistasNutricionistas de España; [citado el 17 de noviembre de 2014]. Disponible en: http://www.consejodietistasnutricionistas. com/

2. Fundación Española de Dietistas-Nutricionistas [portal en internet]. Pamplona: Fundación Española de DietistasNutricionistas; [citado el 17 de noviembre de 2014]. Disponible en: http://www.fedn.es/

3. Consejo General de Dietistas-Nutricionistas de España. Código Deontológico de la profesión de Dietista-Nutricionista [portal en internet]. Valencia: Consejo General de DietistasNutricionistas de España; [citado el 17 de noviembre de 2014]. Disponible en: http://www.consejodietistasnutricionistas. com/wp-content/uploads/2014/06/Codigo-Deontologico-Vs2013-final.pdf

4. Dietética Sin Patrocinadores [portal en internet]. Valencia: Dietética Sin Patrocinadores; [citado el 6 de octubre de 2014]. Disponible en: http://dieteticasinpatrocinadores.org/

5. Orden $\mathrm{CIN} / 730 / 2009$, de 18 de marzo, por la que se establecen los requisitos para la verificación de los títulos universitarios oficiales que habiliten para el ejercicio de la profesión de Dietista-Nutricionista. Boletín Oficial del Estado num.73, del 26-03-2009. Obtenido el 17 de noviembre de 2014 de http://www.boe.es/boe/dias/2009/03/26/pdfs/ BOE-A-2009-5037.pdf

6. Agencia Nacional de Evaluación de la Calidad. ¿Qué estudiar y dónde? [portal en internet]. Madrid: Agencia Nacional de Evaluación de la Calidad; [citado el 6 de octubre de 2014]. Disponible en: http://srv.aneca.es/ListadoTitulos/busquedatitulaciones

7. Universidad Alfonso X el Sabio. Grado en Nutrición Humana y Dietética [portal en internet]. Madrid: Universidad Alfonso X el Sabio; [citado el 6 de octubre de 2014]. Disponible en: http:// www.uax.es/grado-en-nutricion-humana-y-dietetica.html

8. Universidad Alfonso X el Sabio. Doble Grado en Farmacia y Nutrición Humana y Dietética [portal en internet]. Madrid: Universidad Alfonso X el Sabio; [citado el 6 de octubre de 2014]. Disponible en: http://www.uax.es/doble-grado-enfarmacia-y-nutricion-humana-y-dietetica.html

9. Universidad Alfonso X el Sabio. Doble Grado en Ciencias de la
Actividad Física y el Deporte y Nutrición Humana y Dietética [portal en internet]. Madrid: Universidad Alfonso X el Sabio; [citado el 6 de octubre de 2014]. Disponible en: http://www. uax.es/doble-grado-en-ciencias-de-la-actividad-fisica-y-eldeporte-y-nutricion-humana-y-dietetica.html

10. Universidad Autónoma de Madrid. Grado en Nutrición Humana y Dietética [portal en internet]. Madrid: Universidad Autónoma de Madrid; [citado el 6 de octubre de 2014]. Disponible en: https://www.uam.es/ss/Satellite/Ciencias/ es/1242655568847/contenidoFinal/Nutricion_Humana_y_ Dietetica.htm

11. Universidad Católica de Valencia San Vicente Mártir. Doble Grado en Enfermería y Nutrición Humana y Dietética [portal en internet]. Valencia: Universidad Católica de Valencia San Vicente Mártir; [citado el 6 de octubre de 2014]. Disponible en: https://www.ucv.es/estudios_doble_8.asp

12. Universidad Católica San Antonio. Grado en Nutrición Humana y Dietética [portal en internet]. Murcia: Universidad Católica San Antonio; [citado el 6 de octubre de 2014]. Disponible en: http://www.ucam.edu/estudios/grados/nutricion-presencial

13. Universidad Católica San Antonio. Grado en Nutrición Humana y Dietética y Ciencia y Tecnología de los Alimentos [portal en internet]. Murcia: Universidad Católica San Antonio; [citado el 6 de octubre de 2014]. Disponible en: http://www.ucam. edu/estudios/dobles-grados/doble-grado-en-nutricion-ytecnologia-de-alimentos

14. Universidad CEU Cardenal Herrera. Doble Grado en Farmacia y Nutrición Humana y Dietética [portal en internet]. Valencia: Universidad CEU Cardenal Herrera; [citado el 6 de octubre de 2014]. Disponible en: http://www.uchceu.es/estudios/grado/ farmacia_nutricion_dietetica.aspx

15. Universidad CEU San Pablo. Grado en Nutrición Humana y Dietética [portal en internet]. Madrid: Universidad CEU San Pablo; [citado el 6 de octubre de 2014]. Disponible en: http:// www.uspceu.com/es/estudios/grado/farmacia/nutricionhumana-dietetica/presentacion.php

16. Universidad CEU San Pablo. Doble Grado en Farmacia y Nutrición Humana y Dietética [portal en internet]. Madrid: Universidad CEU San Pablo; [citado el 6 de octubre de 2014]. Disponible en: http://www.uspceu.com/es/estudios/ grado/farmacia/farmacia-y-nutricion-humana-y-dietetica/ presentacion.php

17. Universidad CEU San Pablo. Doble Grado en Enfermería y Nutrición Humana y Dietética [portal en internet]. Madrid: Universidad CEU San Pablo; [citado el 6 de octubre de 2014]. Disponible en: http://www.uspceu.com/futuroalumno/pdf/ FARMACIA_14_02.pdf

18. Universidad Complutense de Madrid. Grado en Nutrición Humana y Dietética [portal en internet]. Madrid: Universidad Complutense de Madrid; [citado el 6 de octubre de 2014]. Disponible en: http://www.ucm.es/estudios/gradonutricionhumanaydietetica

19. Universidad de Alicante. Grado en Nutrición Humana y Dietética [portal en internet]. Alicante: Universidad de Alicante; [citado el 6 de octubre de 2014]. Disponible en: http://cvnet.cpd.ua.es/webcvnet/planestudio/planestudiond. aspx? plan $=\mathrm{C} 352$

20. Universidad de Barcelona. Grado de Nutrición Humana y Dietética [portal en internet]. Barcelona: Universidad de 
Barcelona; [citado el 6 de octubre de 2014]. Disponible en: http://www.ub.edu/web/ub/es/estudis/oferta_formativa/ graus/fitxa/N/G1053/index.html

21. Universidad de Granada. Grado en Nutrición Humana y Dietética [portal en internet]. Granada: Universidad de Granada; [citado el 6 de octubre de 2014]. Disponible en: http://grados.ugr.es/nutricion/

22. Universidad de Lleida. Grado en Nutrición Humana y Dietética [portal en internet]. Lleida: Universidad de Lleida; [citado el 6 de octubre de 2014]. Disponible en: http://www.nutricio.udl. cat/es

23. Universidad de Murcia. Grado en Nutrición Humana y Dietética [portal en internet]. Murcia: Universidad de Murcia; [citado el 6 de octubre de 2014]. Disponible en: http://www.um.es/ web/ccsociosanitarias/contenido/estudios/grados/nutricion

24. Universidad de Navarra. Grado en Nutrición Humana y Dietética [portal en internet]. Pamplona: Universidad de Navarra; [citado el 6 de octubre de 2014]. Disponible en: http://www.unav.edu/web/grado-en-nutricion-humana-ydietetica

25. Universidad de Navarra. Doble Grado en Farmacia y Nutrición Humana y Dietética [portal en internet]. Pamplona: Universidad de Navarra; [citado el 6 de octubre de 2014]. Disponible en: http://www.unav.edu/web/doble-grado-enfarmacia-y-nutricion-humana-y-dietetica

26. Universidad Pablo de Olavide. Grado en Nutrición Humana y Dietética [portal en internet]. Sevilla: Universidad Pablo de Olavide; [citado el 6 de octubre de 2014]. Disponible en: http://www.upo.es/portal/impe/web/contenido/0f4b662143b3-11de-874c-3fe5a96f4a88?channel=c1f3624d-2f4711de-b088-3fe5a96f4a88

27. Universidad de Santiago de Compostela. Grado en Nutrición Humana y Dietética [portal en internet]. Santiago de Compostela: Universidad de Santiago de Compostela; [citado el 6 de octubre de 2014]. Disponible en: http://www.usc.es/ es/centros/ciencias/titulacions.html? plan=13947\&estudio $=1$ $3948 \&$ codEstudio $=13550$ \&valor $=9$

28. Universidad de Valencia. Grado en Nutrición Humana y Dietética [portal en internet]. Valencia: Universidad de Valencia; [citado el 6 de octubre de 2014]. Disponible en: http://www.uv.es/uvweb/universitat/es/estudis-grau/grau1285846094474/Titulacio.html?id=1285847387076

29. Universidad de Valencia. Doble Grado en Farmacia y Nutrición
Humana y Dietética [portal en internet]. Valencia: Universidad de Valencia; [citado el 6 de octubre de 2014]. Disponible en: http://www.uv.es/uvweb/universidad/es/estudios-grado/ grau-1285846094474/Titulacio.html?id=1285870306896

30. Universidad de Valladolid. Grado en Nutrición Humana y Dietética [portal en internet]. Valladolid: Universidad de Valladolid; [citado el 6 de octubre de 2014]. Disponible en: http://www.uva.es/export/sites/uva/2.docencia/2.01. grados/2.01.02.ofertaformativagrados/2.01.02.01.alfabetica/ Grado-en-Nutricion-Humana-y-Dietetica/

31. Universidad de Vic. Grado en Nutrición Humana y Dietética [portal en internet]. Vic: Universidad de Vic; [citado el 6 de octubre de 2014]. Disponible en: http://www.uvic.es/es/ estudi/nutricio-humana-i-dietetica

32. Universidad de Zaragoza. Grado en Nutrición Humana y Dietética [portal en internet]. Huesca: Universidad de Zaragoza; [citado el 6 de octubre de 2014]. Disponible en: http://titulaciones.unizar.es/nutricion-humana-dietetica/

33. Universidad del País Vasco. Grado en Nutrición Humana y Dietética[portalen internet].Bizkaia:UniversidaddelPaís Vasco; [citado el 6 de octubre de 2014]. Disponible en: http://www. ehu.es/es/web/vicer.grado-innovacion/aurtengo-graduakarloa?p_auth=sF92mHVG\&p_p_id=upvehuapp_WAR upvehuappportlet\&p_p_lifecycle $=1 \&$ p_p_state $=$ normal $\&$ p_p_ mode $=$ view \&p_p_col_id $=$ column $-2 \& p_{-}$_p_col_count $=1 \&$ _ upvehuapp_WAR_upvehuappportlet_action=redirectAction

34. Universidad Internacional Isabel I de Castilla. Doble titulación de Grado en Ciencias de la Actividad Física y el Deporte y Grado en Nutrición Humana y Dietética [portal en internet]. Burgos: Universidad Internacional Isabel I de Castilla; [citado el 6 de octubre de 2014]. Disponible en: http://www.ui1.es/ dobles-titulaciones/CAFD-NHYD

35. Universidad Ramón Llull. Grado en Nutrición Humana y Dietética [portal en internet]. Barcelona: Universidad Ramón Llull; [citado el 6 de octubre de 2014]. Disponible en: http:// www.blanquerna.edu/es/grado-nutricion-humana-dietetica

36. Universidad Rovira i Virgili. Grado de Nutrición Humana y Dietética [portal en internet]. Tarragona: Universidad Rovira i Virgili; [citado el 6 de octubre de 2014]. Disponible en: http:// www.urv.cat/cae/graus/es_graudnutricio.html

37. Sociedad Española de Medicina de Familia y Comunitaria. Sobre bioética y medicina de familia. Palma de Mallorca: Sociedad Española de Medicina de Familia y Comunitaria; 1996. 